\title{
Variable timestep algorithm for molecular dynamics simulation of non-equilibrium processes
}

\author{
Nigel A. Marks ${ }^{1,2}$, Marc Robinson ${ }^{2}$ \\ ${ }^{1}$ Discipline of Physics and Astronomy, Curtin University, GPO Box U1987, Perth WA 6845, Australia \\ ${ }^{2}$ Nanochemistry Research Institute, Curtin University, GPO Box U1987, Perth WA 6845, Australia
}

\begin{abstract}
A simple, yet robust variable timestep algorithm is developed for use in molecular dynamics simulations of energetic processes. Single-particle Kepler orbits are studied to study the relationship between trajectory properties and the critical timestep for constant integration error. Over a wide variety of conditions the magnitude of the maximum force is found to correlate linearly with the inverse critical timestep. Other quantities used in the literature such as the time derivative of the force and the product of the velocity and force also show reasonable correlations, but not to the same extent. Application of the corresponding metric $\left\|\mathbf{F}_{\max }\right\| \Delta t$ in molecular dynamics simulation of radiation damage in graphite shows that the scheme is both straightforward to implement and effective. In tests on a $1 \mathrm{keV}$ cascade the timestep varies by over two orders of magnitude with minimal loss of energy conservation.
\end{abstract}

\section{Introduction}

Selecting the integration timestep $\Delta t$ in a Molecular Dynamics (MD) simulation is an important yet imprecise exercise. Typically one chooses a value of $\Delta t$ such that the conserved quantity fluctuates by only a "small" amount and does not exhibit linear drift with time. The conserved quantity $\mathrm{E}_{\text {tot }}$ is in turn dependent on both the ensemble employed and the details of any thermostats or barostats; for an NVE simulation $\mathrm{E}_{\text {tot }}$ is simply the sum of the kinetic and potential energy, while for NVT or NPT simulations employing the Nosé-Hoover approach $\mathrm{E}_{\text {tot }}$ is a generalized quantity encompassing the physical and fictitious degrees of freedom. Regardless of the ensemble, one unavoidable aspect of an MD simulation is that $\mathrm{E}_{\text {tot }}$ can never be conserved exactly for finite $\Delta t$, and hence in phase space the computed trajectories will always oscillate to some degree around the true solution. If only on pragmatic grounds, the MD practitioner must come to terms with this unnerving characteristic, albeit supported by the assurance that in many equilibrium situations the discretely integrated trajectories will generate the correct ensemble averages.

The importance of appropriately selecting the timestep comes to the fore in highly non- equilibrium simulations of radiation damage cascades and ion implantation. In such situations the correct value of $\Delta t$ is impossible to determine in advance since the degree of integration precision required will depend on unknown impact parameters for highly energetic encounters. Since using a fixed timestep makes the wallclock time intolerably long, the only practical solution is to employ a variable timestep in which $\Delta t$ is decreased or increased as circumstances dictate. The difficulty then becomes how to choose $\Delta t$ for an arbitrary situation. Historically, a substantial number of empirical variable timestep algorithms have been developed, many of which lie undocumented within in-house codes. The recent article by Stuart et al. [1] provides an excellent summary of the field, and the diverse range of references, several of which are rather obscure, are testament to the non-systematic manner in which variable step algorithms have been developed. Stuart et al. discuss how the general approach has been the development of ad hoc selection rules in which various quantities such as the magnitude of the force $\mathbf{F}$ or its time derivative $\dot{\mathbf{F}}$ are assumed to correlate well with numerical integration errors. They further note that a common approach is to chose the timestep such that $\|\dot{\mathbf{F}}\| \Delta t$

October 13, 2014 
is approximately constant, adding the caveat that in practice it is extremely difficult to find correlations strong enough to allow efficient prediction of the timestep. Their own method differs significantly to most approaches, involving an energy diffusion constant which is specified by the user and controls drift in the conserved quantity. Other metrics employed in the literature include the maximum velocity or the product of the velocity and force [2] or even a combination of the potential and kinetic energy [3]. The MD packages LAMMPS [4] and DL_POLY [5] employ yet another approach in which the user specifies a maximum distance that an atom can move in one timestep and $\Delta t$ is adjusted accordingly. However, a simple thought experiment for a fast head-on collision shows that this approach cannot be automatic since the user will need to make ad-hoc adjustments to the parameter when the incident energy increases.

The present work arose from radiation damage simulations in graphite performed using an in-house MD package implementing the environment dependent interaction potential (EDIP) for carbon $[6,7]$. The results, reported in detail elsewhere [8], were dependent on the availability of a robust variable timestep algorithm that could cope with primary knock-on atoms spanning a wide energy range. Having surveyed the literature it became apparent that there was no simple algorithm and/or routine which enabled the MD user to "set-and-forget" the variable timestep and have confidence that the result was not dependent on empirical parameters and/or functional forms. Our approach was to revisit the very simplest of MD systems, the singleparticle Kepler case, and search for simple correlations which could be exploited in an $N$-body simulation. Having identifed a suitable metric we implemented the algorithm in the EDIP package and immediately found good results. Due to the ease of coding and the conceptual simplicity we anticipate that this approach will be valuable in other situations where energetic particles are modelled using MD.

\section{Methodology}

The single particle simulations were performed with a small MATLAB script using the Verlet integrator. Trajectories were followed in the $(x, y)$ plane using reduced units, employing a central gravitional-type force of magnitude $F=-1 / r^{2}$ directed towards the origin. As shown in Figure 1(a), the initial conditions place the particle at $\left(x_{0}, 0\right)$ with a velocity in the $y$-direction of $\left(0, v_{0}\right)$. The condition for circular motion is met when $v_{0} \sqrt{x_{0}}=1$, with the simplest case being the unit circle for which $x_{0}=v_{0}=1$. Large values of $v_{0}$ exceeding the escape velocity generate hyperbolic orbits, while bound orbits are generally elliptical in shape, with the origin at one of the foci. These elliptical orbits form the primary source of information for this study.

Figure 1(b) shows the time variation in the kinetic, potential and total energy for the elliptical orbit shown in panel (a). The initial conditions are $x_{0}=1$ and $v_{0}=0.9$ and the timestep $\Delta t=0.1$ Three

(a)
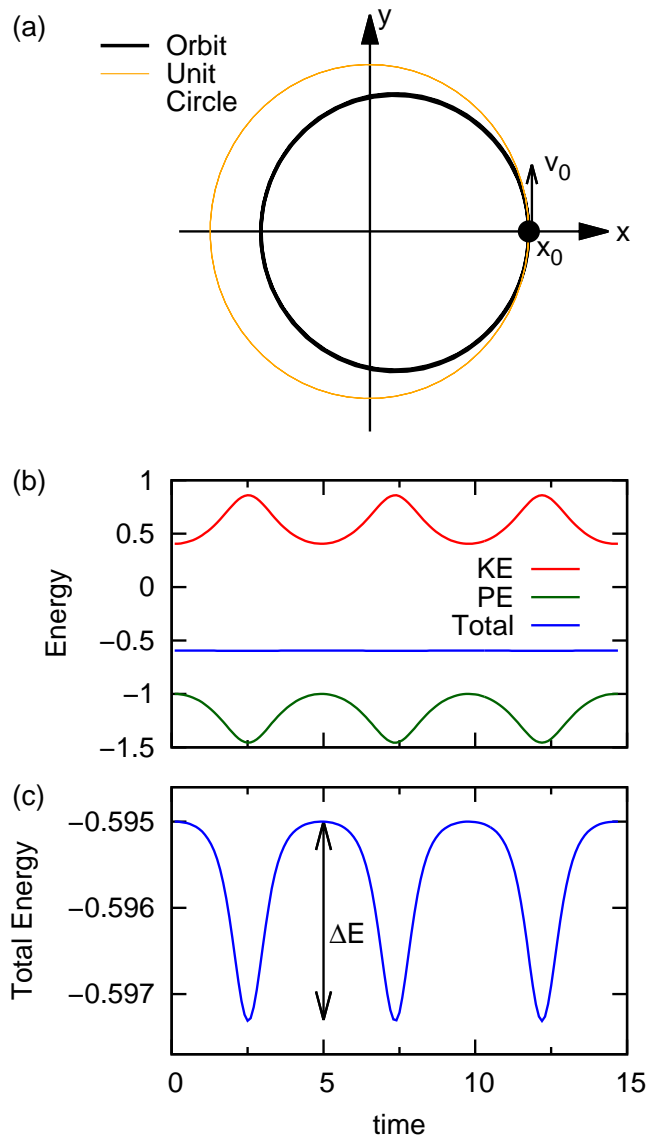

Figure 1: (a) Single particle elliptical trajectory for an object circulating about the origin under an inverse-square force law; the orange line indicates a circular orbit. (b) Potential energy $(\mathrm{PE})$, kinetic energy (KE) and total energy $\left(\mathrm{E}_{\mathrm{tot}}\right)$ as a function of time for the single particle trajectory in (a) with $x_{0}=1, v_{0}=0.9$ and $\Delta t=0.1$. All quantities in reduced units. (c) Close-up view of the total energy ( $\left.E_{\text {tot }}\right)$ in panel (b) demonstrating oscillations of magnitude $\Delta E$. 
orbits are shown, with the kinetic and potential energy exhibiting familiar oscillations, namely the highest kinetic energy and most negative potential energy at perihelion, and vice-versa at aphelion. The most important detail is the total energy $\mathrm{E}_{\mathrm{tot}}$, i.e. the sum of the kinetic and potential energies. In panel (b) $E_{\text {tot }}$ appears to be constant but in fact exhibits small oscillations as shown in Figure 1(c). We quantify these oscillations by defining a quantity $\Delta E$ equal to the maximum variation in $\mathrm{E}_{\text {tot }}$ throughout an orbit, noting that after a complete orbit the instantaneous value of $\mathrm{E}_{\mathrm{tot}}$ has returned to its initial value. One of the central ideas in this work is to generate a range of trajectory sets with different values of $\Delta E$ and to utilize this information to extract a critical timestep corresponding to constant error integration.

The observation in Figure 1(c) that $\mathrm{E}_{\text {tot }}$ is precisely conserved after a complete orbit reflects the symplectic nature of the Verlet integrator; even if the calculation is run for very many orbits the same behaviour remains. This is not the case, however, for non-symplectic integrators such as predictorcorrector methods, which do not exhibit long-term stability and $\mathrm{E}_{\text {tot }}$ drifts over time. Symplectic integrators have long-time stability due to timereversability and conservation of phase-space volume; the latter is difficult to appreciate without reference to the Liouville formulation, but timereversibility can be easily grasped by consideration of the standard Verlet form, in which positions are updated according to the expression:

$$
\mathbf{x}(t+\Delta t)=2 \mathbf{x}(t)-\mathbf{x}(t-\Delta t)+(\Delta t)^{2} \mathbf{a}(t)
$$

and the local error in the position has order $(\Delta t)^{4}$. Determining the velocities is optional using the Verlet method with regards the trajectory, but obviously essential for calculating kinetic energy; here the relevant expression is:

$$
\mathbf{v}(t)=\frac{1}{2 \Delta t}[\mathbf{x}(t+\Delta t)-\mathbf{x}(t-\Delta t)]
$$

and the local error has order $(\Delta t)^{2}$. Accordingly, the local error in the conserved quantity $E_{\text {tot }}$ has order $(\Delta t)^{4}$ since the kinetic energy depends on the velocity-squared and the potential energy is a function of position. One of the most subtle aspects of integrators is how the global error, defined as the accumulated local error over multiple integration steps, varies with time. As noted above, for a symplectic integrator the global error does not linearly increase with time, but within an individual orbit it clearly varies as quantified by $\Delta E$. While in principle it may be possible to analytically derive the global error based on the details of the integrator, trajectory and timestep, we find it more straightforward to employ a numerical approach. Finally, we note that the results are equally applicable to the Velocity-Verlet method as both integrators generate identical trajectories.

\section{Results}

\subsection{Single Particle System}

The starting point for our approach is the underlying relationship between $\Delta E$ and $\Delta t$. Here, the critical link is a power-law dependence as illustrated in Figure 2. Trajectories were generated for a circular orbit with $x_{0}=v_{0}=1$ and an elliptical orbit with $x_{0}=1$ and $v_{0}=0.95$. Ten different values of $\Delta t$ were used, equally spaced betwen 0.01 and 0.1 . The straight line on the log-log plot demonstrates that $\Delta E \propto(\Delta t)^{2}$ for the elliptical orbit and $\Delta E \propto(\Delta t)^{4}$ for the circular case. Regardless of the value of $x_{0}$ or $v_{0}$, elliptical orbits always have a quadratic dependence on the timestep, even when $\Delta t$ is substantially smaller. Only for the special case of circular orbits is the quartic behavior seen. In this work we will make particular use of the quadratic dependence for elliptical orbits, since these trajectories can be considered representative for an arbitrary interaction in an MD simulation, although equally hyperbolic orbits could be employed to achieve the same result.

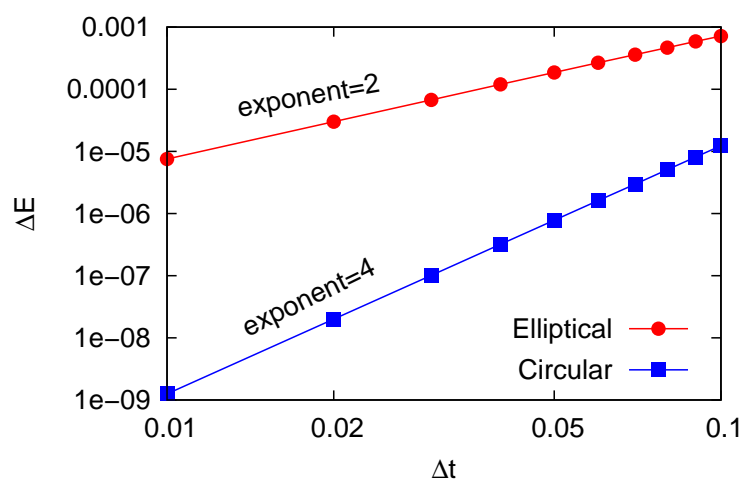

Figure 2: Maximum global error $\Delta E$ as a function of timestep $\Delta t$ for circular orbits (red circles) and elliptical orbits (blue squares) in the Kepler problem of Figure 1. Initial conditions are $\left(x_{0}, v_{0}\right)=(1,1)$ and $\left(x_{0}, v_{0}\right)=(1,0.95)$ respectively. The constant slope indicates that $\Delta E$ varies as the timestep squared for elliptical orbits, and as the fourth power for circular orbits. 
The fact that the maximum global error depends quadratically on the timestep is a fundamental property of the integrator that is by no means obvious since the global error involves sequential addition of small errors which progressively accumulate and cancel throughout the orbit. To test that this behavior is not specific to the Kepler case, we also considered a Hookes Law central force (i.e. $F=-r$ ) which similarly produces closed orbits that are either circular or elliptical depending on the initial conditions. As for the Kepler case, the global error exhibits quadratic and quartic dependency on the timestep for elliptical and circular orbits, respectively. As a final test, we also explored a central force for which the orbits are not closed, but instead precess about the origin. Even for a hypothetical force law of $F=-1 / r^{1.5}$ the relationship between $\Delta E$ and $\Delta t$ remains the same, confirming that the quadratic/quartic behaviour is a fundamental property of the integrator and not tied to any particular detail of the interaction.

Having established that for a general orbit $\Delta E$ depends quadratically on $\Delta t$, we now exploit this property to define a critical timestep $\delta t_{\text {crit }}$ corresponding to integration with constant $\Delta E$. The approach is demonstrated graphically in Figure 3 where $\Delta E$ is plotted for three elliptical orbits and a variety of timesteps; the solid lines are quadratic fits to the data. The horizontal dashed line denotes an arbitrarily chosen integration error, here $1 \times 10^{-3}$, and the vertical lines identify the corresponding value of $\delta t_{\text {crit }}$. It is correspondingly

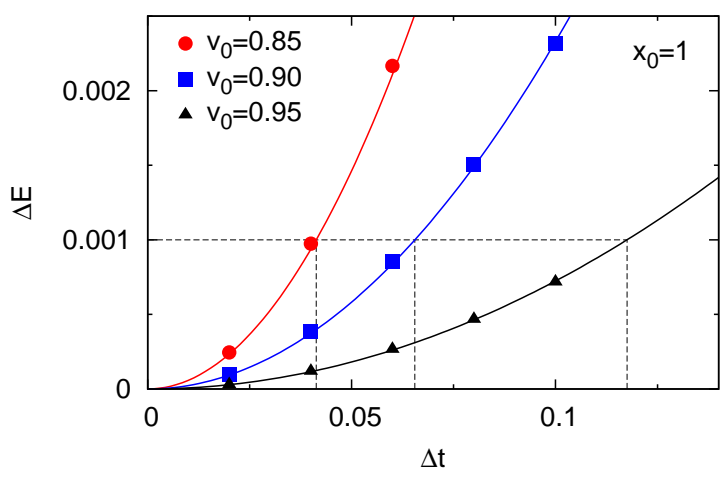

Figure 3: Graphical illustration of the scheme to extract the critical timestep $\delta t_{\text {crit }}$ corresponding to integration with constant global error. Horizontal dashed line indicates the desired error, here chosen as $1 \times 10^{-3}$, while vertical lines denotes the corresponding values of $\delta t_{\text {crit }}$. The solid points represent three different elliptical orbits, all with $x_{0}=1$ and $v_{0}$ as indicated, and the solid lines are quadratic fits.
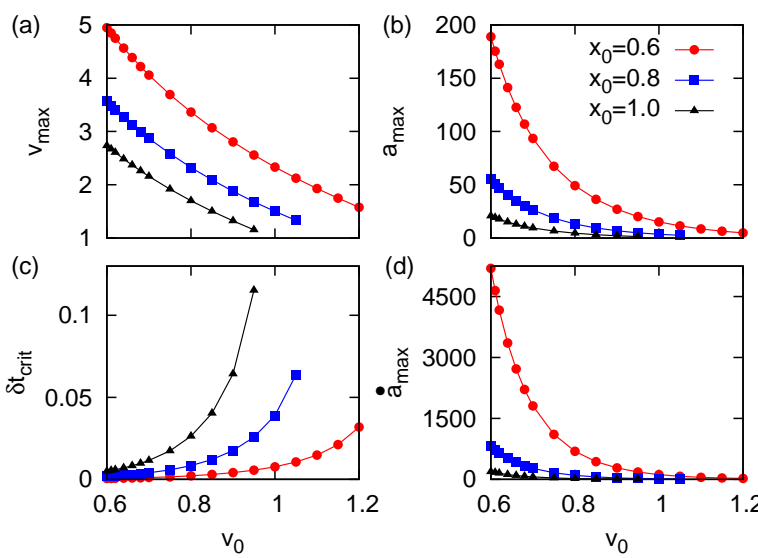

(d)

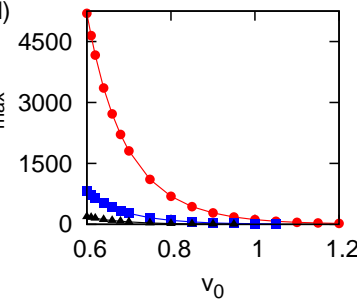

Figure 4: (a) Maximum velocity $v_{\max }$ throughout an orbit for a suite of elliptical trajectories as a function of initial velocity $v_{0}$ and initial position $x_{0}$. (b,d) Same as (a) but for maximum acceleration and its time derivative. (c) Critical timestep $\delta t_{\text {crit }}$ for the indicated trajectories.

straightforward to determine $\delta t_{\text {crit }}$ for an orbit characterized by a particular $\left(x_{0}, v_{0}\right)$ since only a small number of values of $\Delta t$ are required to accurately determine the coefficient of the quadratic function; throughout we use five points. Note that the $\delta t_{\text {crit }}$ values cannot be absolute since they depend on the integration error threshold. In fact, the meaningful quantity is the relative value of $\delta t_{\text {crit }}$ as these ratios are independent of the threshold selected. For clarity we will employ a threshold of $1 \times 10^{-3}$ in the remainder of this manuscript, but the values of $\delta t_{\text {crit }}$ can be easily rescaled for a different threshold using the appropriate multiplicative factor.

The next step in our approach is to simulate a large suite of elliptical trajectories spanning a wide range of parameter space. For each combination of $\left(x_{0}, v_{0}\right)$ the critical timestep is determined and various trajectory parameters are extracted. Examples of the latter include the maximum velocity, $v_{\max }$, the maximum acceleration, $a_{\max }$, and the maximum value of the time deriviative of the acceleration, $\dot{a}_{\max }$. All of these quantities can be easily determined in an MD simulation, and hence any correlation between these quantities and the critical timestep is immensely useful. Figure 4 presents a subset of the entire suite of trajectories, showing the dependence of all four quantities as a function of velocity and for three different initial positions. The general relationship between the various quantities can be easily appreciated, with highly elliptical orbits involving large values of $v_{\max }, a_{\max }$ and 

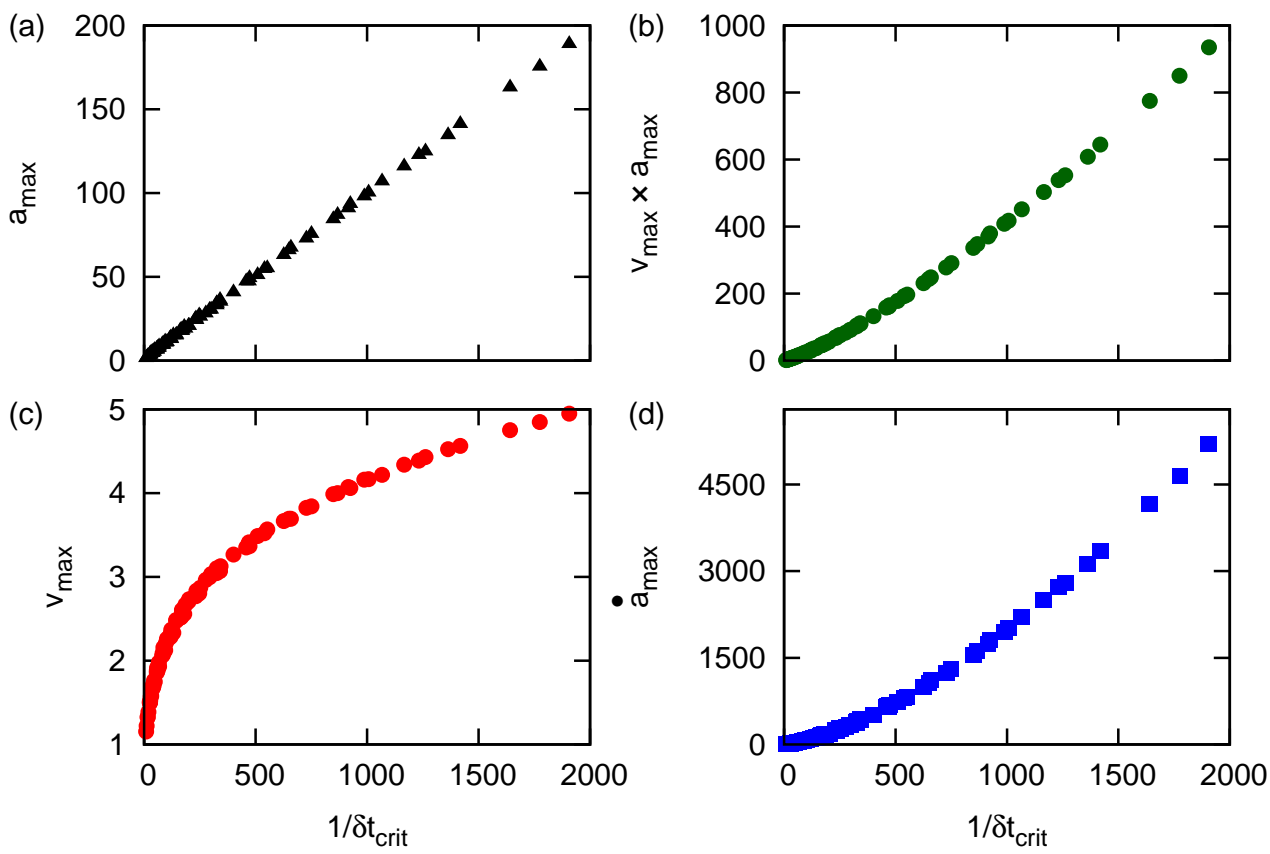

Figure 5: Correlation between various trajectory quantities and the inverse critical timestep $\delta t_{\text {crit }}$ for constant error integration. (a) Maximum acceleration, showing a strong linear variation with inverse timstep, (b) Product of velocity and acceleration, showing a near-linear correlation with inverse timestep (c) Velocity, showing a highly non-linear correlation with inverse timstep, and (d) Time derivative of acceleration, showing a polynomial correlation with inverse timstep.

$\dot{a}_{\max }$ and small critical timesteps, and conversely for orbits with lower eccentricity. Trajectories close to a circular orbit (defined as $\left|v_{0} \sqrt{x_{0}}-1\right|<0.05$ ) were omitted from the data suite, as these points were clear outliers in the search for correlations. In total, the set of initial conditions spanned 19 velocities (all shown in Figure 4) and 6 positions, with $x_{0}=0.65,0.70$ and 0.9 in addition to the three values shown in the figure. These parameters generated a broad data set, with the critical timestep spanning more than two orders of magnitude, and even greater varation in $\dot{a}_{\text {max }}$.

To extract correlations from the data set we explored numerous dependencies of the trajectory quantities on the critical timestep. Since the highly elliptical orbits are the most significant for modelling radiation damage processes, we found it helpful to plot $v_{\max }, a_{\max }$, etc, as a function of the inverse critical timestep as this emphasises the very small timesteps which create the greatest difficulties in a close-approach energetic collision. Four graphs from this analysis are shown in Figure 5, demonstrating the correlation between various trajectory quantities and $1 / \delta t_{\text {crit }}$. It is immediately clear in Figure $5(\mathrm{c})$ that the velocity $v_{\max }$ does not correlate linearly with the inverse timestep which explains why the $\Delta t \propto 1 / v$ algorithm employed in Ref. [2] did not work particularly well. In the same work, the $1 / v$ relation was empirically augmented by a second expression where $\Delta t \propto 1 / F v$, and interestingly, our data provides a post-hoc justification for this choice. In Figure 5(b) we see that the product of maximum velocity and acceleration correlates reasonably well with inverse critical timestep, and while the relationship is not exactly linear, it is sufficiently close to explain why the algorithm used in Ref. [2] was successful. Panel (d) in Figure 5 provides an assessment of the $\|\dot{\mathbf{F}}\| \Delta t$ metric discussed earlier. While there is a reasonable correlation, it is not particularly linear and contains a clear quadratic component which reduces its usefullness for timestep prediction. By far the best correlation is seen in Figure 5(a) where the maximum acceleration shows strong linear correlation with the inverse critical timestep over a wide range of conditions, spanning more than two orders of magnitude. This relationship immediately suggests that the timestep $\Delta t$ in an MD simulation should be inversely proportional to the maximum force, or equivalently, that $\|\mathbf{F}\| \Delta t$ should be approximately constant. 


\section{2. $N$-Body System}

Having identified a suitable relationship between the timestep and trajectory quantities, we tested and applied the algorithm in our MD package implementing the EDIP potential for carbon $[6,7]$. The EDIP approach comprises two-body and threebody interactions mediated by a bond-order-like coordination term. Long-range aspherical terms in the latter allow EDIP to accurately describe the pathway between graphite and diamond, enabling an accurate desription of liquid, amorphous and crystalline forms of carbon, including nanodiamonds [9], nanotubes [10], glassy carbon [11] and carbon onions $[12,13]$. To describe close-approach interactions present in a radiation damage cascade the EDIP potential smoothly switches across to the Ziegler-Biersack-Littmark (ZBL) pair potential [14] as first employed in $[15,16]$ and described in detail in [8]. All calculations were performed in an NVE ensemble and the Verlet integrator was used.

Two categories of testing were performed, a simple two-atom collision with an impact parameter of zero, and a complete simulation of a radiation damage cascade in graphite. Calculations were performed in reduced units, with energies in $\mathrm{eV}$ and lengths in Angstroms. Masses were reduced by the mass of a ${ }^{12} \mathrm{C}$ atom. The cascade simulation contained 88,704 atoms in an orthorhombic supercell measuring approximately $90 \AA$ along each side and initially at $300 \mathrm{~K}$. A primary knock-on atom (PKA) was initiated with an off-axis orientation and an initial kinetic energy of $1 \mathrm{keV}$. The atom selected as PKA was chosen to avoid interactions with the boundaries and with the initial direction oriented towards the centre of the cell. Atomic motions were followed for 5 ps, more than sufficient to capture the cascade due to the high rate of energy transfer to the graphite lattice.

Inspection of the two types of systems quickly established that the product of the force and the timestep was indeed a useful metric for determining $\Delta t$. With the two-atom system it was particularly straightforward to determined that a product of 0.2 resulted in good energy conservation when one atom was directed towards the other with high velocity. As soon as the metric exceeded this value the timestep was halved, and hence the period of high force was automatically associated with a small value of $\Delta t$ and accurate integration followed. For the cascade system it is necessary for the timestep to also increase, and here the timestep was increased by $50 \%$ if the product of the timestep and the maximum force on any atom was below 0.04 for more than 100 steps. This gradual approach to increasing the timestep was adopted to avoid frequent disruptions to the integrator which break its symplectic nature and generate drift in the conserved quantity.

Figure 6 shows a time series of images for the $1 \mathrm{keV}$ cascade, demonstrating the fractal-like character of the trajectories as discussed in detail in [8]. The PKA is initiated in the bottom-right region of Figure 6(a) and is directed towards the topleft corner. At the instant shown in panel (a) the first heavy collision has just occurred and the cascade has split into two sub-cascades. At $t=0.016 \mathrm{ps}$ [Figure 6(b)], the more energetic upper branch is about to undergo further branching, the results of which are more or less complete by $t=0.039$ ps [Figure $6(\mathrm{c})]$. Further evolution of the cascade results in annealing of some transient defects and end-ofrange motion of energetic atoms as shown in the final structure seen in Figure 6(d).

Figure 7 plots the variation in the timestep and the total energy throughout the cascade and provides an instructive demonstration of the algorithm. Each heavy collision results in a sharp drop

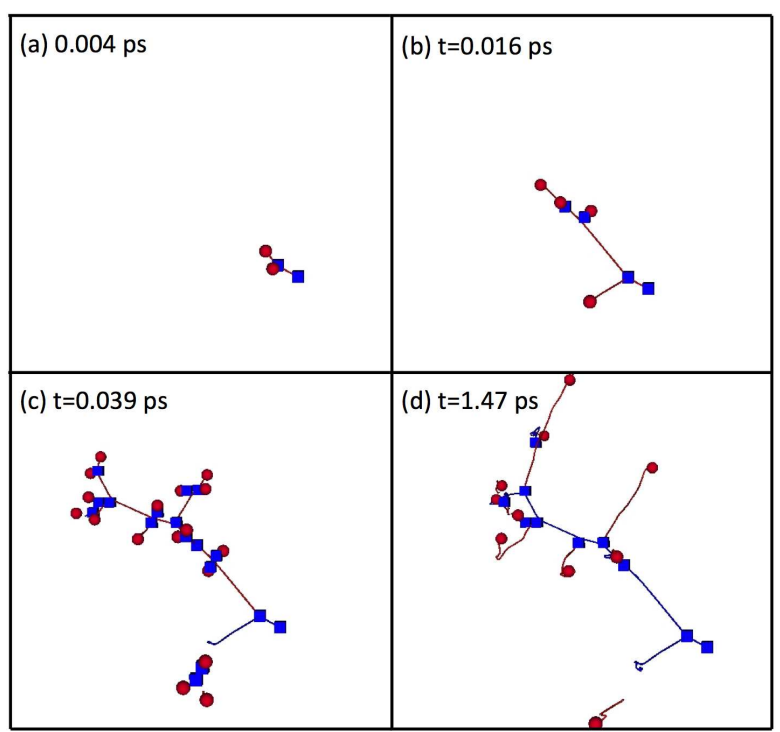

Figure 6: Time evolution of a $1 \mathrm{keV}$ radiation damage cascade in graphite employing the variable timestep algorithm developed in this work. The initial PKA direction is off-axis and orientated towards the top-left of panel (a). Most of the atoms remain on their lattice sites and are not shown. Blue squares and red circles denote vacancy and interstitials determined using a threshold of $0.9 \AA$. The blue and red lines show the associated trajectories of the defects. 

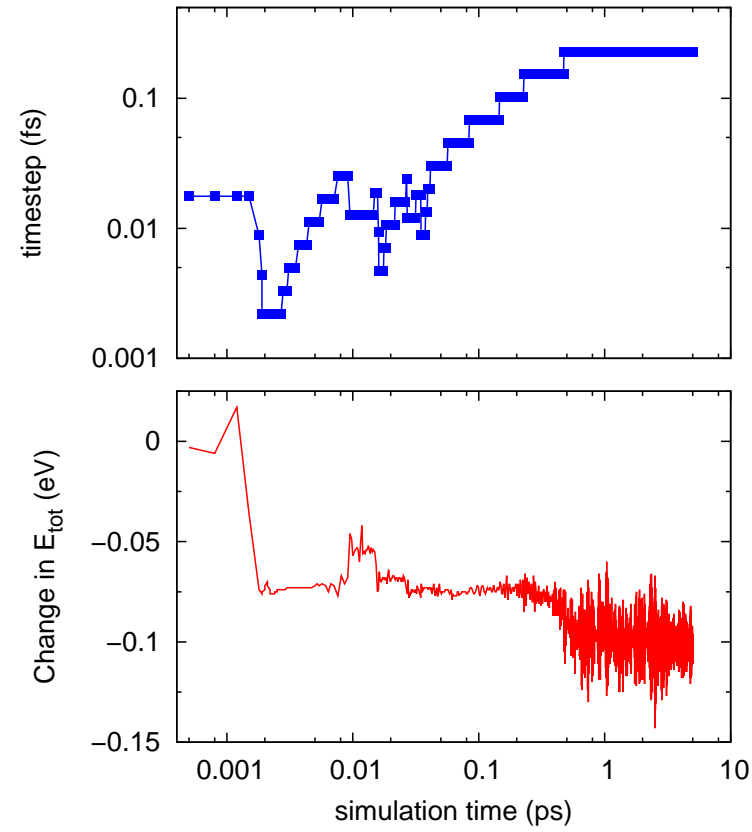

Figure 7: Variation in the timestep and total energy as a function of simulation time for the $1 \mathrm{keV}$ graphite cascade shown in Figure 6. The logarthmic scale on the horizontal axis emphasizes how the timestep cycles down and up multiple times early in the cascade, before trending consistently upwards as thermal equilibrium is reached. The lower panel shown how the conserved quantity, $\mathrm{E}_{\text {tot }}$ varies throughout the cascade, with small shifts occuring during the highly energetic collisions with small impact parameters.

in timestep that can be easily correlated with a branching point in Figure 6. For example, the initial collision at 0.002 ps occurs just moments before the image shown in Figure 6(a) where the two subcascades are initiated. An extremely small timestep of $0.0022 \mathrm{fs}$ is required to integrate this interaction, but once the collision is complete the timestep gradually increases. Subsequently the timestep continues to rise and fall with collisions around times of $0.01,0.02$ and $0.028 \mathrm{ps}$. The collision around $0.01 \mathrm{ps}$ can be inferred by comparing panels (b) and (d) in Figure 6, and the latter collisions are similarly evident in panel (c). Once the kinetic energy has beeen sufficiently dispersed by the branching behaviour, the timestep becomes much larger and plateaus at 0.22 fs. At this point the system is close to thermal equilibrium with a temperature only slightly above $300 \mathrm{~K}$.

The lower panel in Figure 7 shows how the total energy of the system varies throughout the cascade. Initially the total energy is $-645413.850 \mathrm{eV}$, and at the conclusion of the cascade differs by only $0.1 \mathrm{eV}$. Almost all of this change occurs as a result of a minor integration error during the first heavy impact, and even then the change is acceptably small. From $\sim 0.01$ ps onwards the total energy shifts by only small amounts and linear drift is never present. The shift during the first collision during which $\mathrm{E}_{\text {tot }}$ rises and then falls to a new value different to the original is characteristic of a timestep which is slightly too large. This behavior was observed during tests on the two-atom: when the timestep is larger there is a major shift in $\mathrm{E}_{\text {tot }}$ as a result of the collision, while for even larger timestep the system effectively explodes. Viewed with hindsight, this particular simulation could have benefitted from a slightly smaller timestep (and perhaps a transition at an earlier time), but this would require more complex programming that the almost trivially simple threshold-based approach.

Two software improvements immediately present themselves to make superior use of the $\left\|\mathbf{F}_{\max }\right\| \Delta t$ metric and make the energy conservation even better still. The simplest concept provides the MD package with the ability to "rewind" the simulation a small number of timesteps, perhaps five or ten, so that when the metric exceeds the threshold the simulation can be taken back to a slightly earlier state. With such an approach the entire closeapproach trajectory can then be followed with a timestep optimal for the conditions. While in principle straightforward, the downside is the complexity of the coding and the associated memory management, and to a lesser extent the non-linearity of the output. A more elegant approach would involve tracking the metric as a function of time, computing first and perhaps even second derivatives. This would enable forward estimatation of future values of the metric and hence reduction of the timestep before it exceeds the acceptable range.

Looking to other chemical species, there are two useful extensions which would have high utility in MD packages such as LAMMPS or DL_POLY. The first involves finding appropriate critical threshold values for other single-component systems. Though the establishment of these quantities lies beyond the present scope, the ideal situation would be a simple rule suitable for any atomic mass. Given the role of acceleration in the Verlet algorithm, the first possibility to explore is that the thresholds simply scale with the atomic mass. This hypothesis can be tested using both the single-particle Kepler orbits for a variety of reduced masses as well as MD 
simulations of energetic events in materials such as silicon and pure metals. The second extension, involving multi-component species, is more complicated since single-particle Kepler orbits no longer apply. One possibility is to study a two-particle Kepler-type system in which both particles orbit around the centre of mass. Should simple linear relations be observed such as in Figure 5(a), then the reduced mass of the system may well be the appropriate quantity for scaling the thresholds. Such a rule could then easily be tested empirically on largescale MD systems as for the single-species case. Finally, we note that since multi-component systems contain $\frac{1}{2} s(s+1)$ combinations (where $s$ is the number of species), the least agressive (i.e. most conservative) constraint on $\Delta t$ would need be adopted for the entire system.

\section{Summary}

A method for estimating the timestep in a molecular dynamics (MD) simulation employing Verlettype integration has been developed. Using the observation that the maximum global error varies quadratically with the timestep, single particle Kepler orbits are used to develop the concept of a critical timestep $\delta t_{\text {crit }}$ corresponding to constant integration error. A large suite of elliptical orbits are used to identify correlations between the critical timestep and quantities which can be extracted from an MD trajectory. The maximum force during an orbit is found to correlate linearly with the inverse critical timestep, superior to another relationships used in the MD literature to estimate the timestep, such as velocity, the force-velocity product and the time derivative of the acceleration. Implementation of the variable timestep metric $\left\|\mathbf{F}_{\max }\right\| \Delta t$ in an $\mathrm{MD}$ package leads to a simple and effective scheme for describing radiation damage in graphite. With minimal coding complexity, the timestep is varied up and down over a range spanning two orders of magnitude, enabling accurate simulations of cascade evolution and minimal shifts in the conserved quantity. Such an algorithm and can be easily implemented in commonly used packages. Looking beyond the present work, further improvements can be envisaged in which the time derivative of the metric is calculated by finite difference to anticipate a close encounter and the determination of suitable thresholds for particles of arbitrary mass.

\section{Acknowledgments}

The project used advanced computational resources provided by the iVEC facility at Murdoch University. NAM is supported by an Australian Research Council Future Fellowship (FT120100924). MR thanks the Australian Nuclear Science and Technology Organisation for support.

\section{References}

[1] S. J. Stuart, J. M. Hicks, M. T. Mury, An Iterative Variable-timestep Algorithm for Molecular Dynamics Simulations, Mol. Sim. 29 (3) (2003) 177-186.

[2] K. Nordlund, Molecular dynamics simulations of ion ranges in the $1-100 \mathrm{keV}$ energy range, Comp. Mater. Sci. 3 (1995) 448-456.

[3] R. Smith, private communication in relation to the $L O B M D$ molecular dynamics package developed and applied at Loughborough University, UK.

[4] S. Plimpton, Fast parallel algorithms for shortrange molecular dynamics, J. Comput. Phys. 117 (http://lammps.sandia.gov) (1995) 1-42.

[5] I. T. Todorov, W. Smith, K. Trachenko, M. T. Dove, DL_POLY_3: new dimensions in molecular dynamics simulations via massive parallelism, J. Mater. Chem. 16 (20) (2006) 1911.

[6] N. A. Marks, Generalizing the environment-dependent interaction potential for carbon, Phys. Rev. B 63 (3) (2001) 035401.

[7] N. Marks, Modelling diamond-like carbon with the environment-dependent interaction potential, J. Phys.: Condens. Mat. 14 (11) (2002) 2901-2927.

[8] H. J. Christie, M. Robinson, D. L. Roach, D. K. Ross, I. Suarez-Martinez, N. A. Marks, Simulating Radiation Damage Cascades in Graphite, Carbon, submitted.

[9] N. A. Marks, M. Lattemann, D. R. McKenzie, Nonequilibrium Route to Nanodiamond with Astrophysical Implications, Phys. Rev. Lett. 108 (7) (2012) 075503.

[10] I. Suarez-Martinez, P. J. Higginbottom, N. A. Marks, Molecular dynamics simulations of the transformation of carbon peapods into double-walled carbon nanotubes, Carbon 48 (12) (2010) 3592-3598.

[11] I. Suarez-Martinez, N. A. Marks, Effect of microstructure on the thermal conductivity of disordered carbon, Appl. Phys. Lett. 99 (3) (2011) 033101.

[12] D. W. M. Lau, D. G. McCulloch, N. A. Marks, N. R. Madsen, A. V. Rode, High-temperature formation of concentric fullerene-like structures within foam-like carbon: Experiment and molecular dynamics simulation, Phys. Rev. B 75 (23) (2007) 233408.

[13] R. C. Powles, N. A. Marks, D. W. M. Lau, Self-assembly of $\mathrm{sp}^{2}$-bonded carbon nanostructures from amorphous precursors, Phys. Rev. B 79 (2009) 075430.

[14] J. F. Ziegler, J. P. Biersack, U. Littmark, The Stopping and Range of Ions in Solids, Vol. 1, Pergamon, New York, 1985.

[15] N. A. Marks, J. M. Bell, G. K. Pearce, D. R. McKenzie, M. M. M. Bilek, Atomistic simulation of energy and temperature effects in the deposition and implantation of amorphous carbon thin films, Diam. Relat. Mater. 12 (10-11) (2003) 2003-2010.

[16] G. K. Pearce, N. A. Marks, D. R. McKenzie, M. M. M. 
Bilek, Molecular dynamics simulation of the thermal spike in amorphous carbon thin films, Diam. Relat. Mater. 14 (3-7) (2005) 921-927. 